\title{
Corrigendum: All-optical bit storage in a fibre laser by optomechanically bound states of solitons
}

M. Pang, W. He, X. Jiang and P. St. J. Russell

Nature Photonics 10, 454-458 (2016); published online 30 May 2016; corrected after print 20 October 2016.

In the version of this Letter originally published, in the Acknowledgements, the name 'G. Onishchukov' was misspelt. This error has now been corrected in the online versions of the Letter. 